\title{
Research on Sensitive Measuring Points for Vibration Fault of NC Turret Based on Energy Flow and Transfer Path
}

\author{
Fei Chen, ${ }^{1}$ DeCong Zhang, ${ }^{2}$ Wei Luo $\left(\mathbb{D},{ }^{3}\right.$ and Binbin $X u^{1}$ \\ ${ }^{1}$ Sino-German College of Intelligent Manufacturing, Shenzhen Technology University, Shenzhen 518118, China \\ ${ }^{2}$ Chang Guang Satellite Technology Co., Ltd., Changchun, China \\ ${ }^{3}$ Key Laboratory of CNC Equipment Reliability, Ministry of Education, School of Mechanical and Aerospace Engineering, \\ Jilin University, Changchun, Jilin 130022, China \\ Correspondence should be addressed to Wei Luo; luoweicn@jlu.edu.cn
}

Received 1 September 2020; Revised 17 December 2020; Accepted 4 February 2021; Published 22 February 2021

Academic Editor: Gordon Huang

Copyright (c) 2021 Fei Chen et al. This is an open access article distributed under the Creative Commons Attribution License, which permits unrestricted use, distribution, and reproduction in any medium, provided the original work is properly cited.

\begin{abstract}
Some equipment fault measurement points are difficult to determine. Thus, this study proposes a method using energy flow and transfer path to select sensitive measurement points. Firstly, based on the energy flow theory of the shell, the energy flow is taken as the physical quantity to describe the energy transfer of the structure vibration, and the energy-based transfer path analysis method is established. Secondly, the simulation model of the finite element energy flow was established by focusing on the NC turret, and the vector image of the energy flow transfer path of the NC turret shell was created. For the relationship between the vector images of the experimental and simulation energy flows, the validity of the finite element energy flow simulation results is verified. Lastly, three abnormal areas of the NC turret shell are identified by comparing and analyzing the energy flow vector images of the NC turret in the healthy and fault states, taking the poor meshing fault of the grooves as an example. By choosing nine test points in the abnormal areas and calculating the distribution rate of the transfer paths between the test points to the joint surfaces of the fluted disc, the energy flow distribution rate of the different fault positions to the test points is obtained. Calculation results enabled us to identify three measuring points that can accurately distinguish the fault location for a typical fault mode. This research combined energy flow theory and transfer path analysis method to determine the vibration fault sensitive measuring points of NC equipment. The proposed method is practical in the field of fault diagnosis and has broad application prospects.
\end{abstract}

\section{Introduction}

At present, sensor tests serve as a major method to obtain equipment vibration data. A reasonable vibration sensor position can extensively monitor the status of equipment and is also the basis of accurate fault diagnosis. However, a mature theoretical research on the location of sensors has yet to be conducted. The fault-related monitoring data, which are available for equipment with complex structures and long mean time between failures, are extremely limited. This current situation substantially increases the difficulty of fault diagnosis technology research. Therefore, the application of energy flow theory in transfer path analysis will provide a new idea for equipment fault diagnosis.

In vibration systems, the essence of vibration transmission is energy transmission. Vibration energy flow theory presents the vibration state of a structure. Energy flow theory can be categorized into five types according to different analysis methods: statistical energy analysis $[1,2]$, traveling wave analysis and wave control [3], structural sound intensity method [4, 5], admittance energy flow method [6,7], and finite element energy flow method [8, 9]. Chen et al. [10] proposed an energy flow model to predict the high-frequency vibration response of a panel in supersonic flow and quantified the effect of supersonic flow on the high-frequency forced vibration characteristics. Oh et al. [11] established a simulation model of a wheel loader based on a MATLAB/Simulink environment, which was used to analyze the energy flow and efficiency of the equipment during the design phase. In summary, the application of energy flow research focuses on identifying load vibration sources and suppressing vibration and noise [12]. 
The transfer path analysis method, based on the linear superposition principle of the linear time-invariant system, tracks several transfer paths in a vibration system. This method is widely used in troubleshooting noise, vibration, and internal load estimation [13]. Zhao et al. [14] provided an effective method based on matrix calculus, generalized second-moment technique, and stochastic finite element theory for the vibration transfer path system to transfer reliability. Janssens et al. [15] proposed a new transfer path analysis method, which can use a simple model for fast fault removal or utilize a complex model, to improve accuracy based on a few measurements.

In recent years, experts have applied energy flow theory to solve transfer path-related problems. Li et al. [16] used the admittance energy flow method as a basis to analyze the vibration transmission characteristics of a double-half-axis turntable. They identified the main vibration source and main vibration transfer path using finite element energy flow theory. Huang et al. [17] analyzed the contribution of the six transfer paths of the fault signal of the wind turbine gearbox and determined the main transfer path by using the finite element energy flow method, which provides a reference for revealing the fault mechanism and feature extraction of the wind turbine gearbox. In summary, energy flow and its visualization technology can directly describe the energy distribution characteristics of the vibration system, particularly for comparing and analyzing the influence caused by path parameter change on energy flow transmission characteristics. Some research methods on fault location have been introduced recently $[18,19]$, and the combination of power flow theory and transfer path analysis method is a new direction in the field of fault diagnosis and has broad application prospects.

The current study presented a method based on finite element energy flow and transfer path to determine vibration fault sensitive points. Based on the energy flow theory of the shell, the energy flow transfer path vector image of NC turret is drawn, and the simulation results of finite element energy flow are verified by energy flow testing. By comparing and analyzing the energy flow vector image of the NC turret in the healthy and failure states, the abnormal areas of vibration energy, which vary substantially in the failure state of the NC turret, were determined. Lastly, the location of the sensitive detection point corresponding to the typical fault mode was determined by calculating the distribution rate of each transfer path amongst the test points.

\section{Analytical Method of Energy Flow and Transfer Path}

2.1. Research on the Energy Flow of Shell. The definition of energy flow represents the ability of external forces to do work or dissipate energy in a structure per unit of time. This physical quantity is crucial in describing the transmission of vibration energy in a structure and can play a critical guiding significance for the research on equipment fault diagnosis.

Steady-state energy flow is calculated as follows:

$$
\begin{aligned}
P & =\frac{1}{T} \int_{0}^{T}|\vec{F}| \cdot|\vec{V}| \cdot \cos \omega t \cdot \cos (\omega t+\phi) \cdot \mathrm{d} t \\
& =-\pi f|\vec{F}| \cdot|\vec{V}| \cdot \sin \phi .
\end{aligned}
$$

Formula (1) shows that calculating the energy flow of a particular node entails knowing the force and velocity (or displacement) responses at that node. The force response can be obtained from the stress at the point, and the stress and velocity (or displacement) of each node can be obtained from the postprocessing of the finite element calculation. Thus, the energy flow of any point in a structure can be obtained.

For shell structures, the vibration forms include longitudinal vibrations in plane and bending, both of which will cause independent deformation. The energy flow at a node for shell structures can be regarded as the superposition of the energy flow of the planar film and shell elements.

For the bending problem of the shell, an element is taken out from the shell to analyze the forces acting on it. The length, width, and height of the element are $\mathrm{d} x, \mathrm{~d} y$, and $\delta$, respectively. As shown in Figures 1 and 2, five stress components act on the cross section: $\sigma_{x}, \sigma_{y}, \tau_{x y}, \tau_{x z}$, and $\tau_{y z}$. Given that $\sigma_{x}$ and $\tau_{x y}$ are proportional to $Z$ and their values are zero in the midplane, their principal vectors of the shell are equal to zero. The shell only produces bending moments, torque $M_{x}, M_{y}, M_{x y}$, and $M_{y x}$, and shear $Q_{x}$ and $Q_{y}$, as shown in formulas (2) and (3):

$$
\begin{aligned}
& \left\{\begin{array}{l}
M_{x}=\int_{-\delta / 2}^{\delta / 2} \sigma_{x} z \mathrm{~d} z \\
M_{y}=\int_{-\delta / 2}^{\delta / 2} \sigma_{y} z \mathrm{~d} z, \\
M_{x y}=M_{y x}=\int_{-\delta / 2}^{\delta / 2} \tau_{x y} z \mathrm{~d} z,
\end{array}\right. \\
& \left\{\begin{array}{l}
Q_{x}=\int_{-\delta / 2}^{\delta / 2} \tau_{x z} \mathrm{~d} z \\
Q_{y}=\int_{-\delta / 2}^{\delta / 2} \tau_{y z} \mathrm{~d} z .
\end{array}\right.
\end{aligned}
$$

For the plane problem, the cross-sectional internal forces of the shell element are expressed in terms of the membrane forces as follows:

$$
\left\{\begin{array}{l}
N_{x}=\int_{-\delta / 2}^{\delta / 2} \sigma_{x} \mathrm{~d} z \\
N_{y}=\int_{-\delta / 2}^{\delta / 2} \sigma_{y} \mathrm{~d} z \\
N_{x y}=\int_{-\delta / 2}^{\delta / 2} \tau_{x y} \mathrm{~d} z
\end{array}\right.
$$

Stress acting on the shell is completely transformed into the internal force acting on its middle surface. By importing formulas (2)-(4) into the steady-state energy flow calculation formula (1), the energy flow expression (5) for a twodimensional shell element can be obtained [20] as follows: 


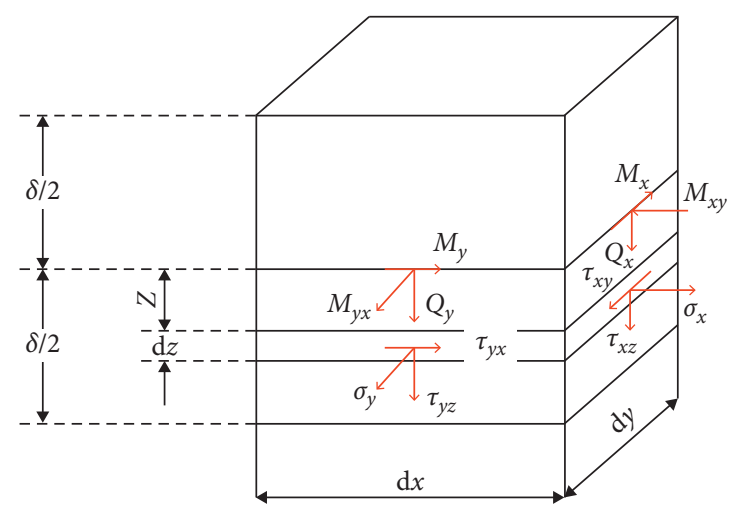

Figure 1: Stress of the shell element.

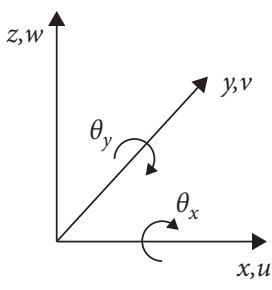

(a)

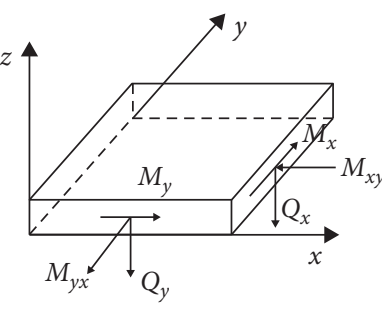

(b)

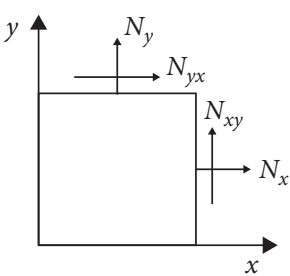

(c)

Figure 2: Internal and cross-sectional forces of the shell element.

$$
\left\{\begin{array}{l}
P_{x}=-\pi f\left(\left|\overrightarrow{N_{x}}\right||\vec{u}|+\left|\overrightarrow{N_{x y}}\right||\vec{v}|+\left|\overrightarrow{Q_{x}}\right||\vec{w}|+\left|\overrightarrow{M_{x}}\right|\left|\overrightarrow{\theta_{y}}\right|+\left|\overrightarrow{M_{x y}}\right|\left|\overrightarrow{\theta_{x}}\right|\right) \sin \phi, \\
P_{y}=-\pi f\left(\left|\overrightarrow{N_{y}}\right||\vec{u}|+\left|\overrightarrow{N_{y x}}\right||\vec{v}|+\left|\overrightarrow{Q_{y}}\right||\vec{w}|+\left|\overrightarrow{M_{y}}\right|\left|\overrightarrow{\theta_{y}}\right|+\left|\overrightarrow{M_{y x}}\right|\left|\overrightarrow{\theta_{y}}\right|\right) \sin \phi,
\end{array}\right.
$$

where $P_{x}$ is the energy flow in the $x$-direction, $P_{y}$ is the energy flow in the $y$-direction, $u$ is the deformation in the $x$ direction, $v$ is the deformation in the $y$-direction, $w$ is the deformation in the $z$-direction, $\theta_{x}$ and $\theta_{y}$ are the angles of rotation, $f$ is the vibration frequency, and $\phi$ is the phase difference.

Thus, the expression for the shell energy flow is as follows:

$$
P=P_{x} i+P_{y} j
$$

2.2. Transfer Path Analysis Based on Energy Flow. In a real vibration system, the external excitation and vibration response are connected by each transfer path of the system. The nature of each transfer path is the vibration transfer characteristic of the vibration system. The vibration transmission characteristics of the system serve as the main links in the analysis of the vibration system to directly determine the dynamic characteristics of the system response [21].

When analyzing the transfer path, the system is assumed to be a linear steady-state vibration system, which satisfies the superposition principle. The vibration of the system vibration source is transmitted from each path to the target point in the form of force and motion. Additionally, the total vibration energy of the target point is the superposition of the energy transferred to the target point along each path.

The basic principle of the energy-based transfer path analysis is as follows:

$$
E_{0}=\sum_{i=1}^{n} \xi_{i} \cdot E_{i}=\xi_{I} \cdot E_{I},
$$

where $E$ is the energy and $\xi$ is the energy-keeping factor.

Energy flow, as a physical quantity describing the vibration energy transfer in the structure, is equivalent to the concept of energy in formula (7). Thus, the total energy flow $P_{o}$ is as follows:

$$
P_{0}=\sum_{i=1}^{n} \xi_{i} \cdot P_{i}=\xi_{I} \cdot P_{I},
$$

where $P_{o}$ is the total energy flow of the target point; $P_{i}$ is the energy flow of transfer path $i$ at the excitation point; $P_{I}=$ $\left[P_{1}, P_{2}, \ldots, P_{n}\right]^{T}$ is the energy flow matrix of each transfer path at the excitation point; $\xi_{i}$ is the energy-keeping factor of 
the transfer path $i$; and $\xi=\left[\xi_{1}, \xi_{2}, \ldots, \xi_{n}\right]^{T}$ is the energykeeping factor matrix for each transfer path.

If the contribution rate was used to express the proportion of the energy of each coupling point in the target point, then the contribution rate of the transfer path $i$ is as follows:

$$
\varepsilon_{i}=\frac{\xi_{i} \cdot P_{i}}{\sum_{i=1}^{n} \xi_{i} \cdot P_{i}} \times 100 \% .
$$

From the single excitation point system (Figure 3), the vibration energy of the excitation point is transferred along each transfer path to the coupling point and converges thereafter to the corresponding target point. According to the transfer path $i$, the path energy-keeping factor of the active subsystem side is $\xi_{i}^{A}$ and the path energy-keeping factor of the passive subsystem side is $\xi_{i}^{B}$. Thereafter, the energy-keeping factor $\xi_{i}$ for the entire path is as follows:

$$
\xi_{i}=\xi_{i}^{A} \cdot \xi_{i}^{B}
$$

In the single excitation point system shown in Figure 3, the energy flow for each transfer path is generally different at the excitation point, thereby resulting in difficulty obtaining the energy flow of each transfer path. To address the current issue, the energy flow at the coupling point and path energykeeping factor in the passive subsystem can be used to obtain the final solution.

$$
\begin{aligned}
P_{i}^{A} & =\xi_{i}^{A} \cdot P_{i}, \\
P_{i}^{B} & =\xi_{i}^{B} \cdot P_{i}^{A}, \\
\varepsilon_{i} & =\frac{\xi_{i}^{B} \cdot P_{i}^{A}}{\sum_{i=1}^{n} \xi_{i}^{B} \cdot P_{i}^{A}} \times 100 \%,
\end{aligned}
$$

where $P_{i}^{A}$ is the energy flow at the coupling point, $P_{\dot{j}}^{B}$ is the energy flow of a transfer path at the target point, $\xi_{i}^{B}$ is the energy retention factor of the passive subsystem, and $\varepsilon_{i}$ is the contribution rate of the transfer path, thereby indicating the proportion of the energy flow of each transfer path at the target point.

In the case of a coupling point corresponding to multiple target points (Figure 4), the proportion of energy received by the passive subsystem to each transfer path is different at the coupling point. The distribution rate represents the proportion of energy transferred to each target point at the coupling point. Therefore, if energy flow at the coupling point and energy-keeping factor of each transfer path of the passive subsystem are already known, then the energy flow distribution rate of the coupling point to different target points can be calculated as follows:

$$
\omega_{j}=\frac{\xi_{j}^{B} \cdot P_{i}^{A}}{\sum_{j=1}^{n} \xi_{j}^{B} \cdot P_{i}^{A}} \times 100 \%,
$$

where $\omega_{j}$ is the distribution rate of the transfer path, representing the proportion of energy flow transferred from the coupling point to different target points, and $\xi_{j}^{B}$ is the energykeeping factor of each transfer path of the passive subsystem.

\section{Research on Finite Element Energy Flow of the NC Turret}

3.1. Research on Finite Element Energy Flow Simulation of the NC Turret. The finite element energy flow method is a technique used to study the energy flow of an element by separating the entire structure with the aid of the finite element concept. Given the maturity of finite element theory, the energy flow of each node can be obtained by achieving the response of each node with finite element software.

Firstly, the $3 \mathrm{D}$ model of the NC turret in CATIA is simplified. The details for appropriate simplification can be disregarded on the premise that the efficiency and accuracy of the analysis results will not be affected. The simplified model of the entire machine is shown in Figure 5. Thereafter, the finite element model is processed in HyperMesh, including mesh generation, joint surface processing, and material assignment. Secondly, the model can be imported into ABAQUS to define the boundary conditions and load dynamic cutting forces. The cutting force with an amplitude value of $200 \mathrm{~N}$ and frequency of $50 \mathrm{~Hz}$ is set as harmonic excitation to be applied to the cutter rod. Finally, the harmonic response of the model is solved in ABAQUS.

From the postprocessing of harmonic response analysis, the stress and displacement components of each node of the NC turret shell are obtained.

They are imported into MATLAB from Excel and drew the vector image of the vibration energy flow of the NC cutter shell with MAYLAB vector drawing tool (Figures 6-9).

As shown in Figures 6 and 7, the energy transfer paths on the two sides of the NC turret shell are not completely the same owing to the dynamic cutting force acting on the right side of the entire NC turret. Moreover, Figure 10 shows that the acting force transmitted to the main part of the NC turret shell by the cutting force can be decomposed into a superposition of a force along the direction of the dynamic cutting force and a torque in the clockwise direction. The torque will cause the opposite trend of vibration energy and transfer to the left and right sides of the NC turret. The part of the energy indicated by the red arrow in Figures 6 and 7 is relatively large, which is mainly caused by the thin wall close to the vibration source (i.e., joint surface of the outer gear disc and the shell) of the NC turret shell. It is noted also that its energy is concentrated.

As shown in Figure 8, harmonic excitation is applied to the simulated cutter rod at the upper left corner of the diagram. The dynamic force's direction is from the horizontal component to the lower right corner of the diagram. Therefore, the overall trend of energy flow is from upper left to lower right. At the point indicated by the red arrow, a cylindrical cavity (i.e., working cavity of the transmitter) with thin shell is present, thereby indicating energy concentration. 


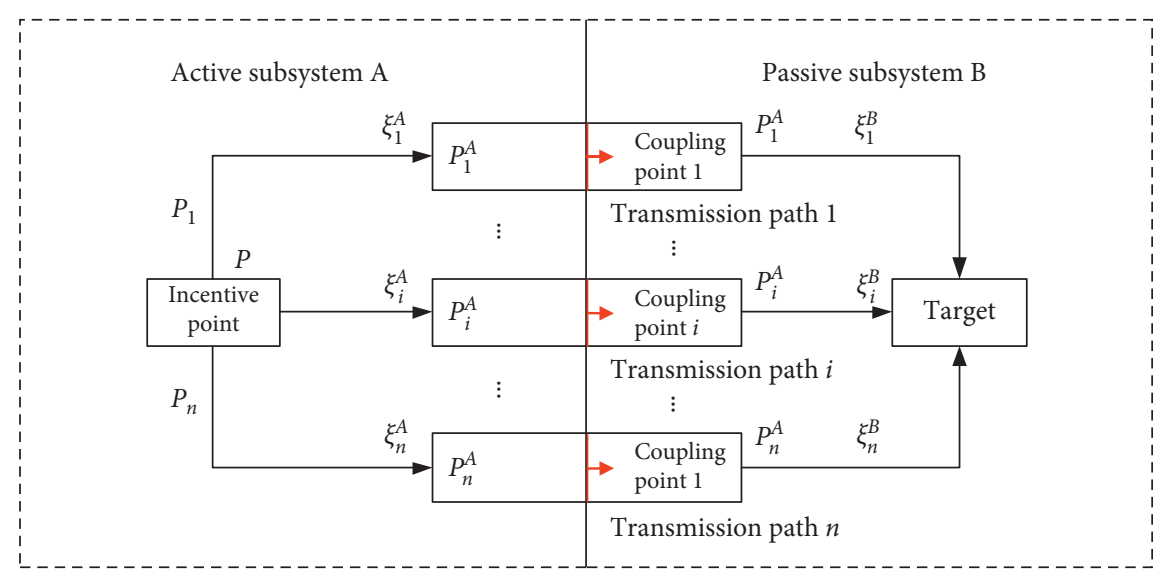

FIgURE 3: Energy transmission of the system.

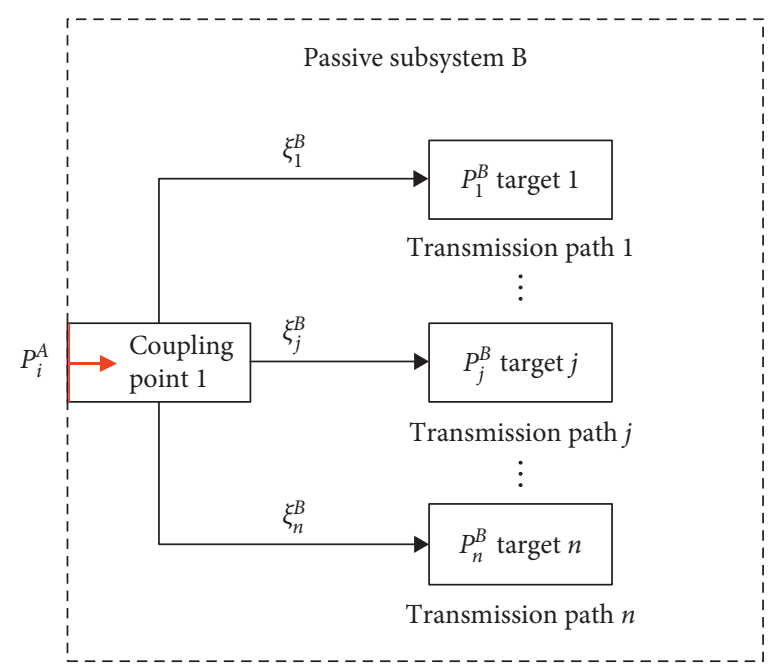

FIGURE 4: Energy transmission of the multitarget and passive subsystems.

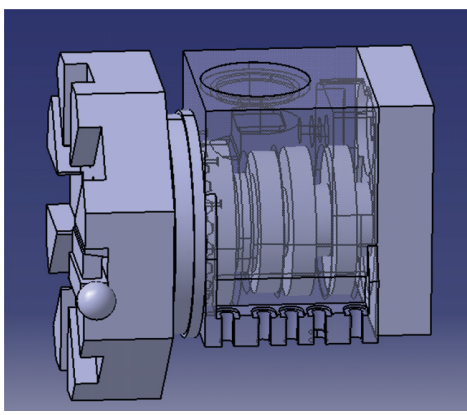

(a)

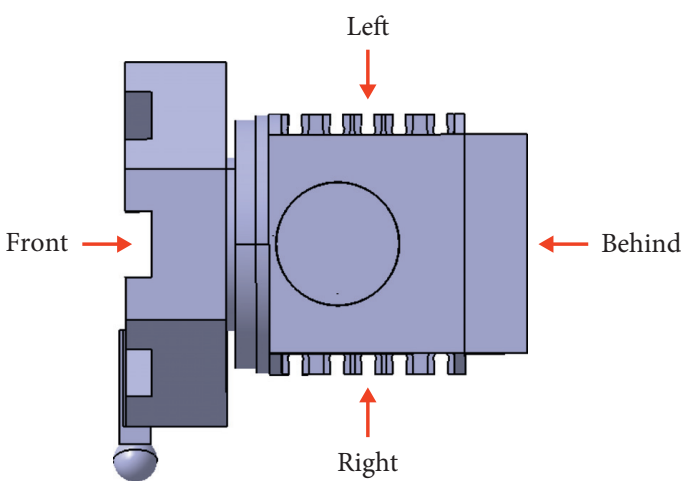

(b)

FIgUre 5: Simplified model of the NC turret.

3.2. Experimental Study on the Energy Flow of the NC Turret. The finite element energy flow test platform for the NC turret is shown in Figure 11. This platform is mainly composed of the NC turret, loading mechanism, IPC, NC turret control cabinet, acquisition system, three-direction acceleration sensor, and supporting device of the corresponding equipment.

In this experiment, the dynamic cutting force with a mean value of $690 \mathrm{~N}$, an amplitude value of $200 \mathrm{~N}$, and a frequency of $50 \mathrm{~Hz}$ is selected to load the simulated cutter 

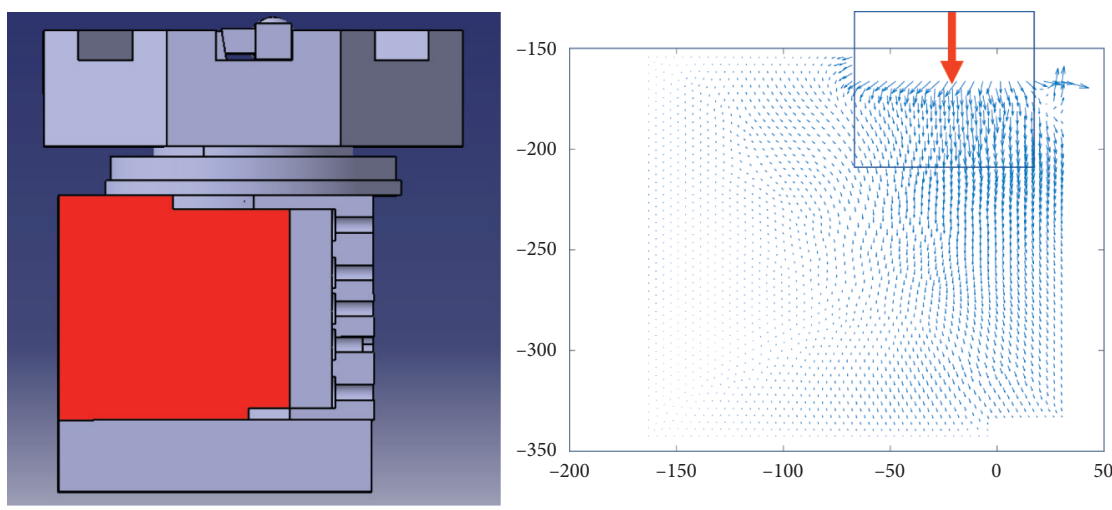

(a)

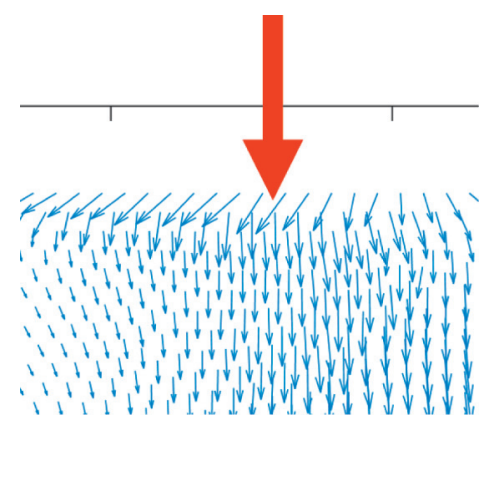

(b)

Figure 6: Vector image of the left side of the NC turret shell and its energy flow.

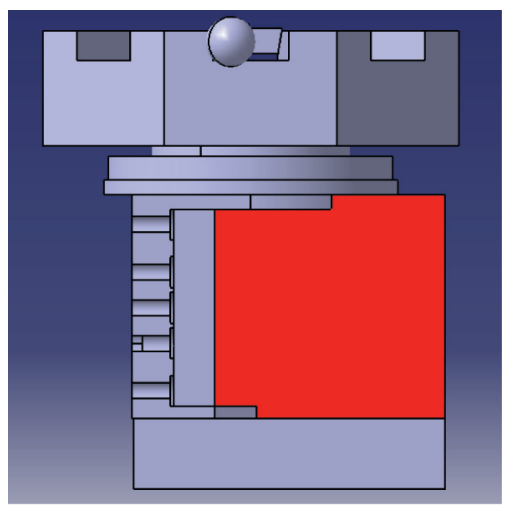

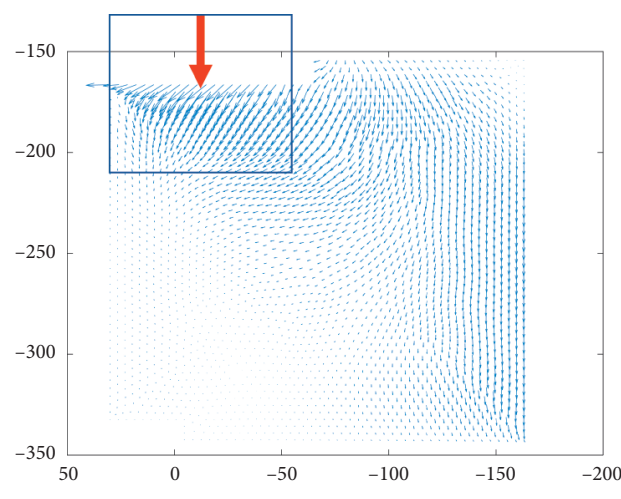

(a)

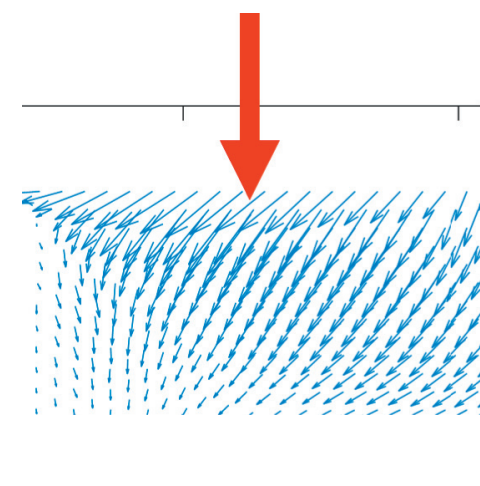

(b)

Figure 7: Vector image of the right side of the NC turret shell and its energy flow.
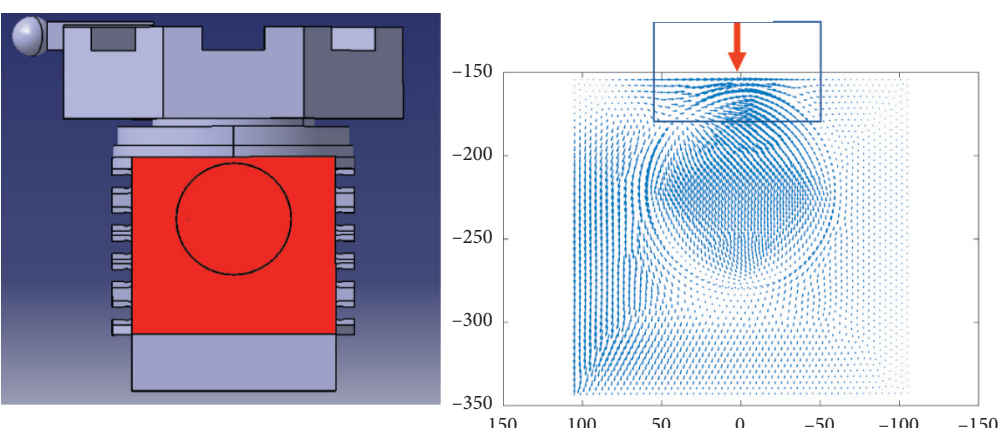

(a)

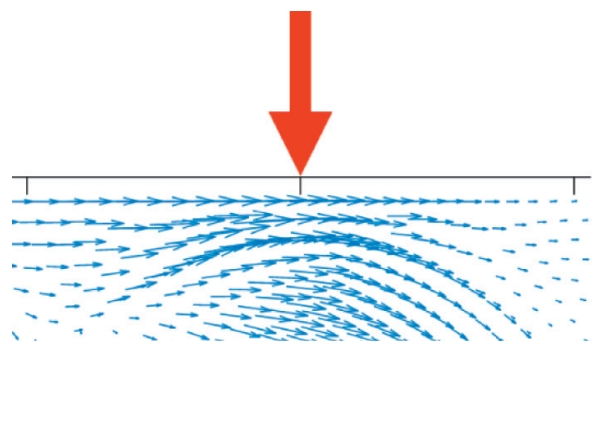

(b)

Figure 8: Vector image of the upper surface of the NC turret shell and its energy flow.

rod. On the side of the NC turret shell, 31 nodes on the side of the NC turret shell which are easy to measure are selected for measurement. The measuring sequence of the nodes is shown in Figure 12. The layout scheme of the sensor is shown in Figure 13, and the acceleration signal acquisition system under a dynamic cutting force load is shown in Figure 14.
By measuring the acceleration values in the $x$ - and $y$ directions, the cross-spectral density using the Welch method in MATLAB is obtained. The cross-spectral density values of 31 measuring nodes in the $x$ - and $y$-directions are recorded. Thereafter, the energy flow of all the measuring nodes in the $x$-and $y$-directions is obtained according to the conversion formula of cross-spectral density and energy flow 

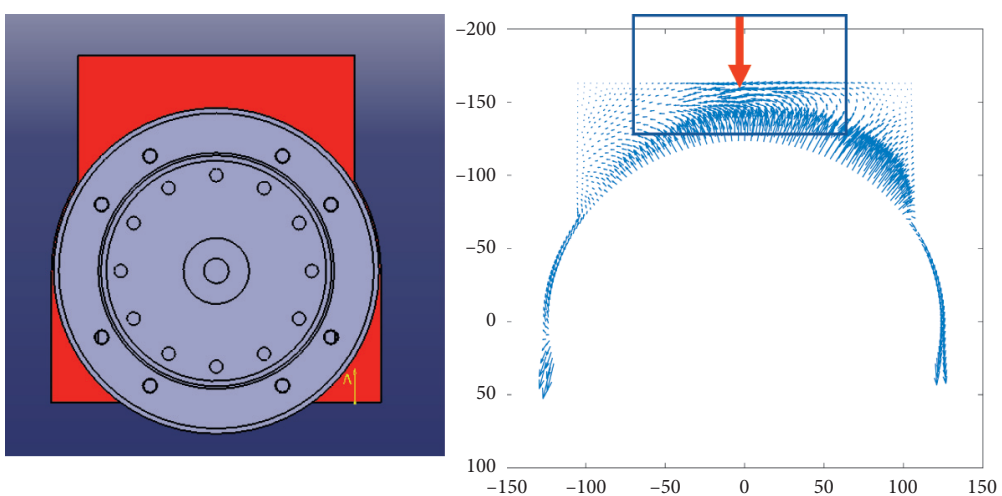

(a)

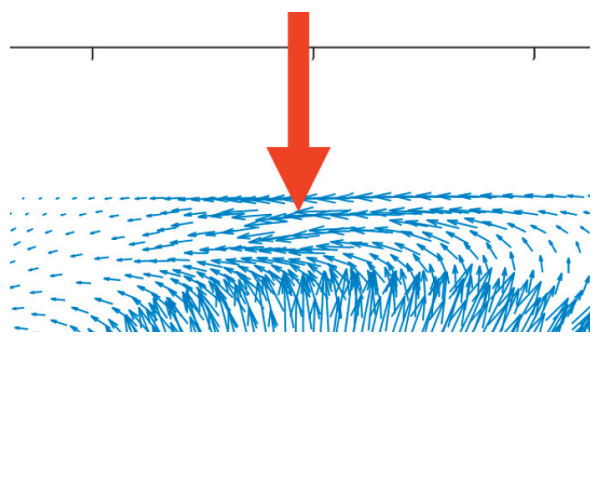

(b)

Figure 9: Vector image of the front surface of the NC turret shell and its energy flow.

in [22]. The energy flow vector images of the NC turret shell are shown in Figures 15 and 16.

Comparing Figures 15 and 16, it can be seen that the energy flow direction of the majority of the measuring nodes approximates that of the simulation. Additionally, the trend of the entire energy flow direction close to the simulation verifies the validity of the finite element energy flow simulation results.

\section{Research on the Sensitive Area of the Vibration Fault of the NC Turret}

As one of the key functional components of the NC turret, the reliability of a fluted disc directly affects the positioning accuracy, repeated positioning accuracy, and the stability of the NC turret. The failures of fluted disc mainly include wear, spalling, corrosion, and other types of surface damage. All these faults will lead to poor contact or damage to the fluted disc's surface when it is engaged.

This study uses the research on the poor contact of fluted disc as a basis to simulate the response status of the NC turret under dynamic cutting force. Therefore, by deleting some constraints of the contact between the fluted disc's teeth in the finite element model of the NC turret, the harmonic response analysis is carried out to simulate the poor contact between the external and locking fluted discs when the NC turret is subjected to the dynamic cutting force. With the application of the finite element energy flow method, the energy flow's vector image of the NC turret shell in the fault state is drawn and compared with the energy flow's vector image in the normal state. Accordingly, the abnormal region of the vibration energy of the NC turret shell in the fault state is determined.

The fluted disc has 24 teeth, and 3 adjacent teeth were divided into a group. Harmonic response analysis was performed on the 8 faulty areas. The schematic of the faulty areas' division is shown in Figure 17, and the stress cloud diagram of the fault simulation result of Zone 1 is shown in Figure 18. The stress nephogram of area 1's fault simulation result is shown in Figure 18.
As shown in Figure 18, the disappearance of the corresponding stress in this region is due to the removal of the restraint between the external and locking fluted discs. However, the fluted disc engages normally in other regions, and the simulation result can evidently express the fault of poor meshing between teeth in some areas. The comparison between the normal status of the NC turret and the energy flow vector image of the fluted disc's poor meshing is shown in Table 1.

Table 1 shows that the energy flow of the left side of the NC turret shell has no evident change owing to the long distance from the fault position. As shown in Figure (d), the energy flow indicated by the red arrow changes its direction and becomes large evidently. On the front surface of the NC turret shell, the energy flow in the right half of Figure (f) is different from that in Figure (e). The energy flow indicated by the black arrow becomes small, whilst the energy flow indicated by the green arrow becomes large and has a tendency to incline to the right side, where energy is minimal.

According to the principle and process mentioned above, a simulated analysis is carried out for areas 2 to 8 . Owing to space constraints, enumerating the eight analysis results is no longer necessary. By summarizing the comparative results of the eight areas, the areas where the vibration energy varies substantially under the poor meshing of the fluted disc are shown in Figure 19.

\section{Research on Vibration Sensitive Measuring Points of the CNC Equipment}

Suitable sensor position can considerably improve the accuracy of equipment fault diagnosis. Based on energy flow theory and transfer path analysis, this paper conducted research on the vibration sensitive measuring points of the $\mathrm{CNC}$ equipment and eventually identified them.

The four vibration transfer paths from the cutter rod to the NC turret shell are as follows: 


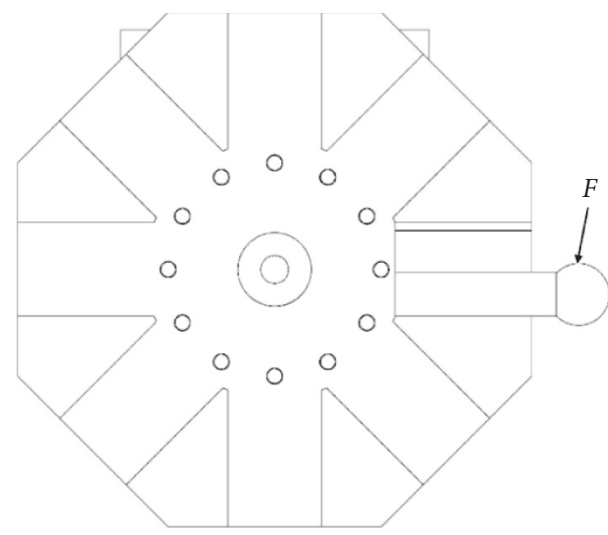

(a)

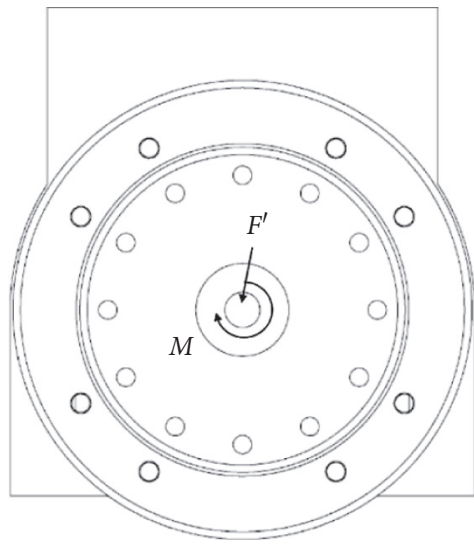

(b)

Figure 10: Force diagram of the NC turret.

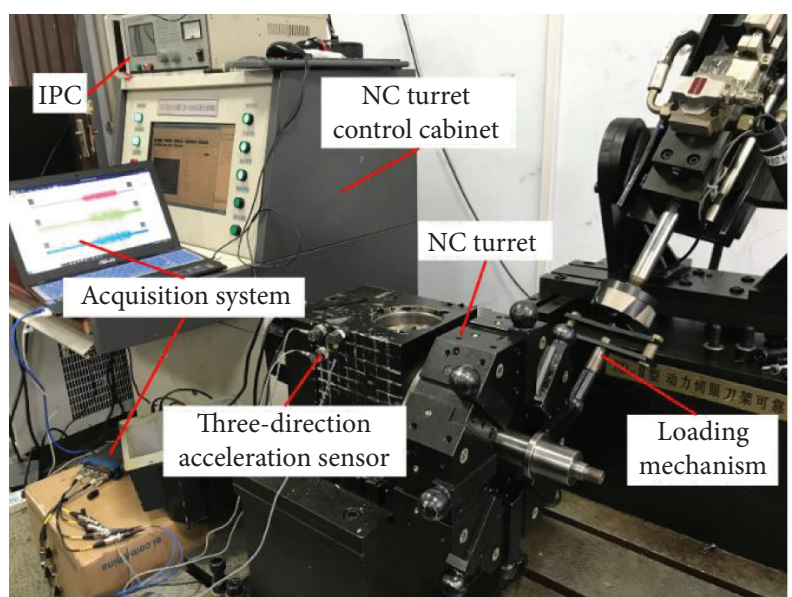

FIGURE 11: Finite element energy flow test platform of the NC turret.

\begin{tabular}{|l|l|l|l|l|l|l|l|l|}
\hline & 1 & 2 & 3 & 4 & 5 & 6 & & \\
\hline & 7 & 8 & 9 & 10 & 11 & 12 & & \\
\hline & 13 & 14 & 15 & 16 & 17 & 18 & & \\
\hline & 19 & & 20 & 21 & 22 & 23 & & \\
\hline & 24 & 25 & 26 & 27 & 28 & 29 & & \\
\hline & 30 & & & & & & & \\
\hline & 31 & & & & & & & \\
\hline
\end{tabular}

FIgURE 12: Measuring sequence of the energy flow test nodes. 


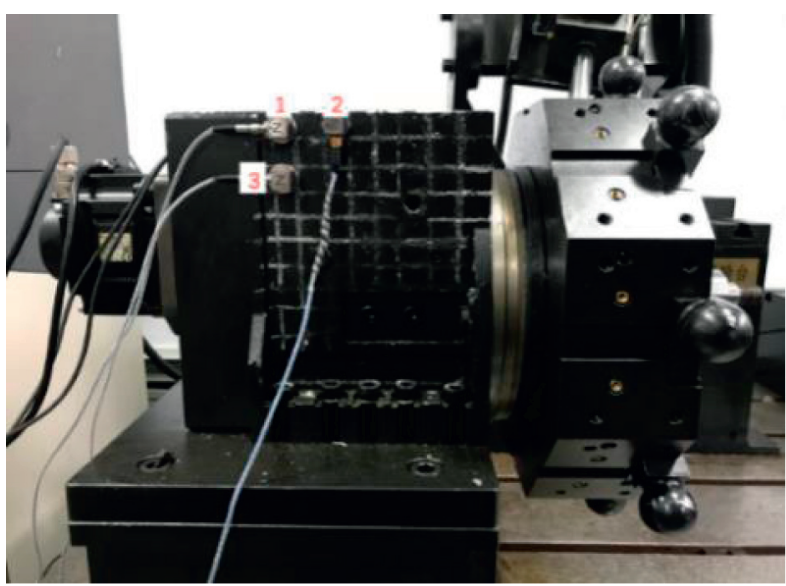

FIGURE 13: Layout scheme of the sensor.

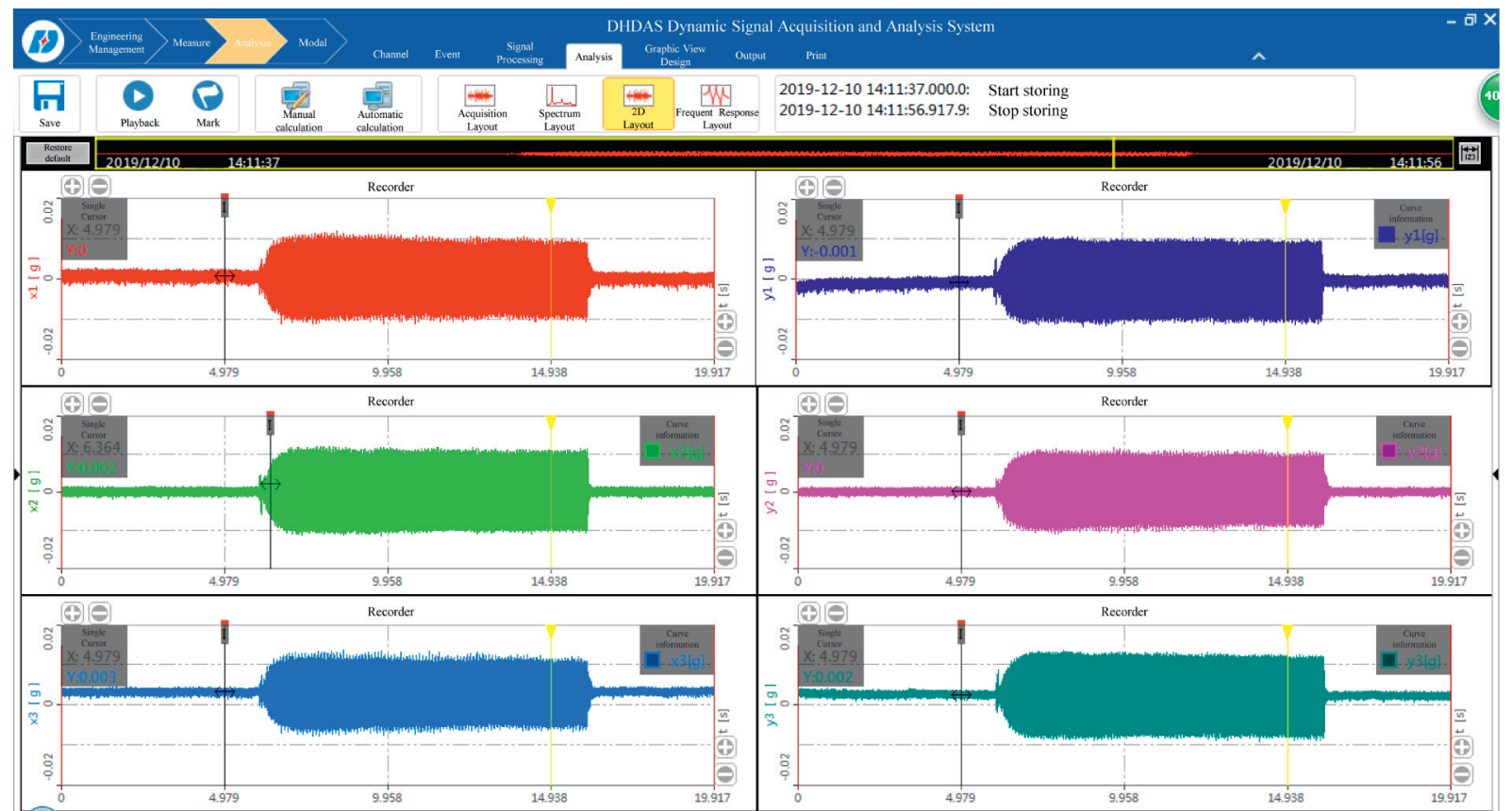

FIgURE 14: Acquisition system of the acceleration signal under a dynamic cutting force load.

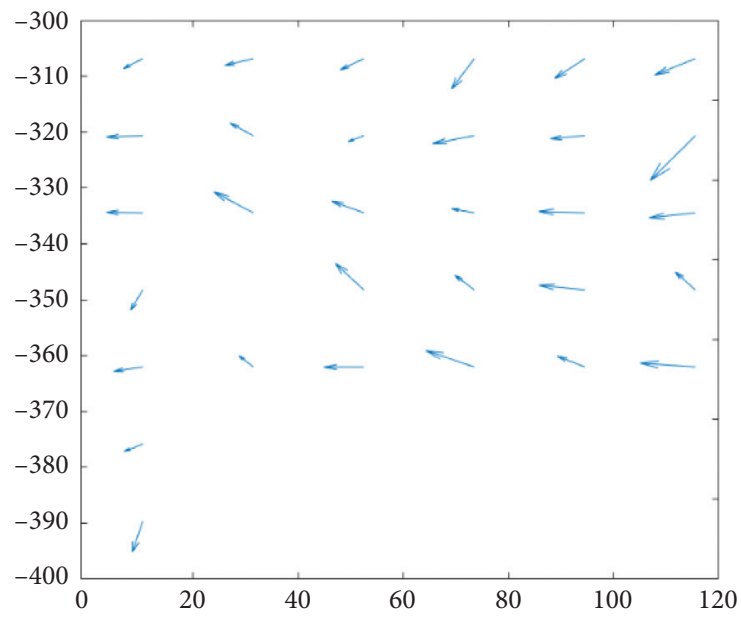

FIGURE 15: Vector image of the experimental energy flow. 


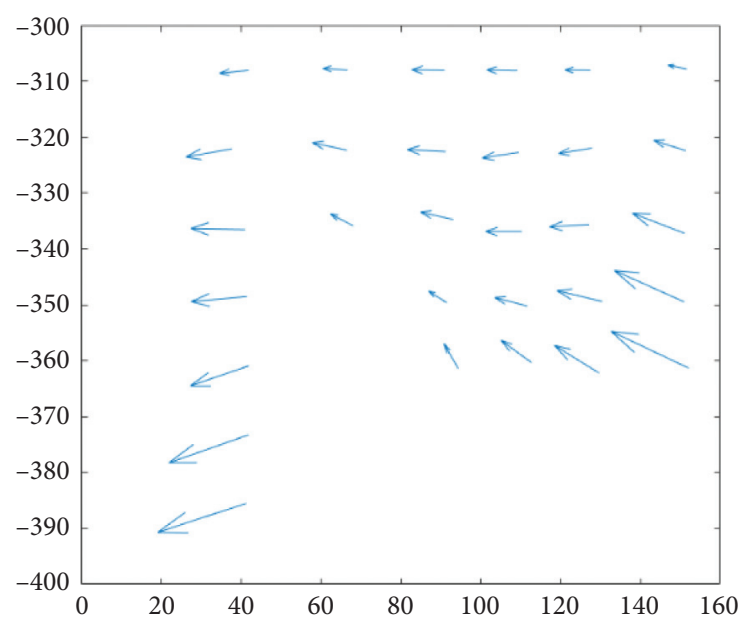

Figure 16: Vector image of the simulation energy flow.

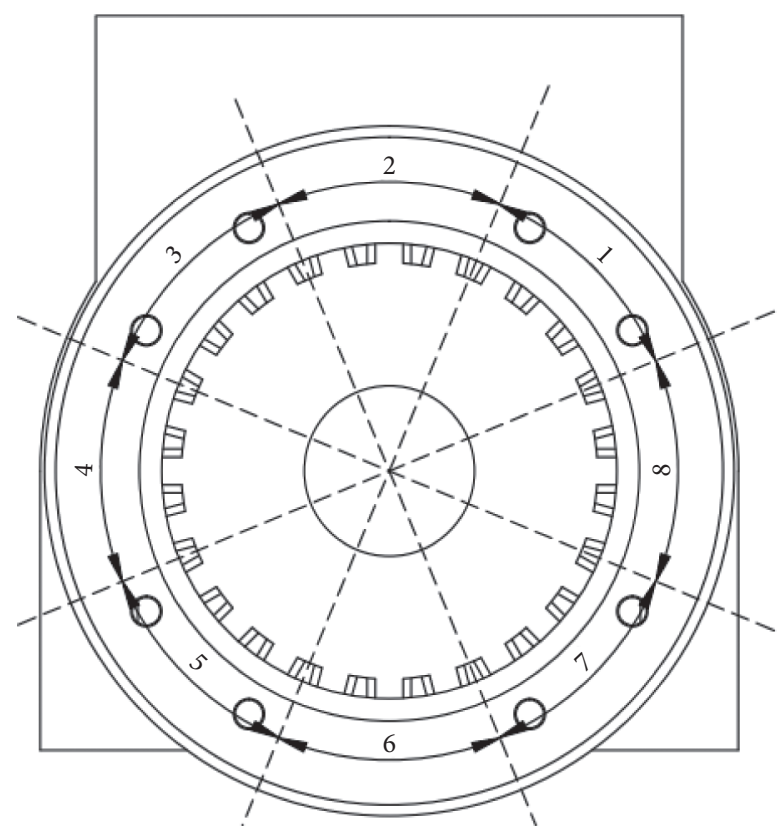

FIgURE 17: Faulty areas' division scheme.

Path 1: Cutter rod $\longrightarrow$ Cutter holder $\longrightarrow$ Cutter head $\longrightarrow$ Internal fluted disc $\longrightarrow$ Locking fluted dis$\mathrm{c} \longrightarrow$ External fluted disc $\longrightarrow$ NC turret shell

Path 2: Cutter rod $\longrightarrow$ Cutter holder $\longrightarrow$ Cutter head $\longrightarrow$ Internal fluted disc $\longrightarrow$ Locking fluted dis$\mathrm{c} \longrightarrow$ Loosening and locking transmission device $\longrightarrow$ NC turret shell

Path 3: Cutter rod $\longrightarrow$ Cutter holder $\longrightarrow$ Cutter head $\longrightarrow$ Internal fluted disc $\longrightarrow$ Spindle $\longrightarrow$ Gear train $\longrightarrow$ bearing $\longrightarrow$ NC turret shell
Path 4: Cutter rod $\longrightarrow$ Cutter holder $\longrightarrow$ Cutter head $\longrightarrow$ Internal fluted disc $\longrightarrow$ Spindle $\longrightarrow$ Bearing $\longrightarrow$ Motor connecting plate $\longrightarrow$ NC turret shell

For path 1, the joint surfaces of the parts are tightly connected by bolts or hydraulic pressure under the normal condition of the NC turret. The loss of vibration energy between the joint surfaces is minimal, and the NC turret shell absorbs the majority of the vibration energy. For path 2, the vibration energy transferred to the NC turret shell is extremely minimal because the connection between the 

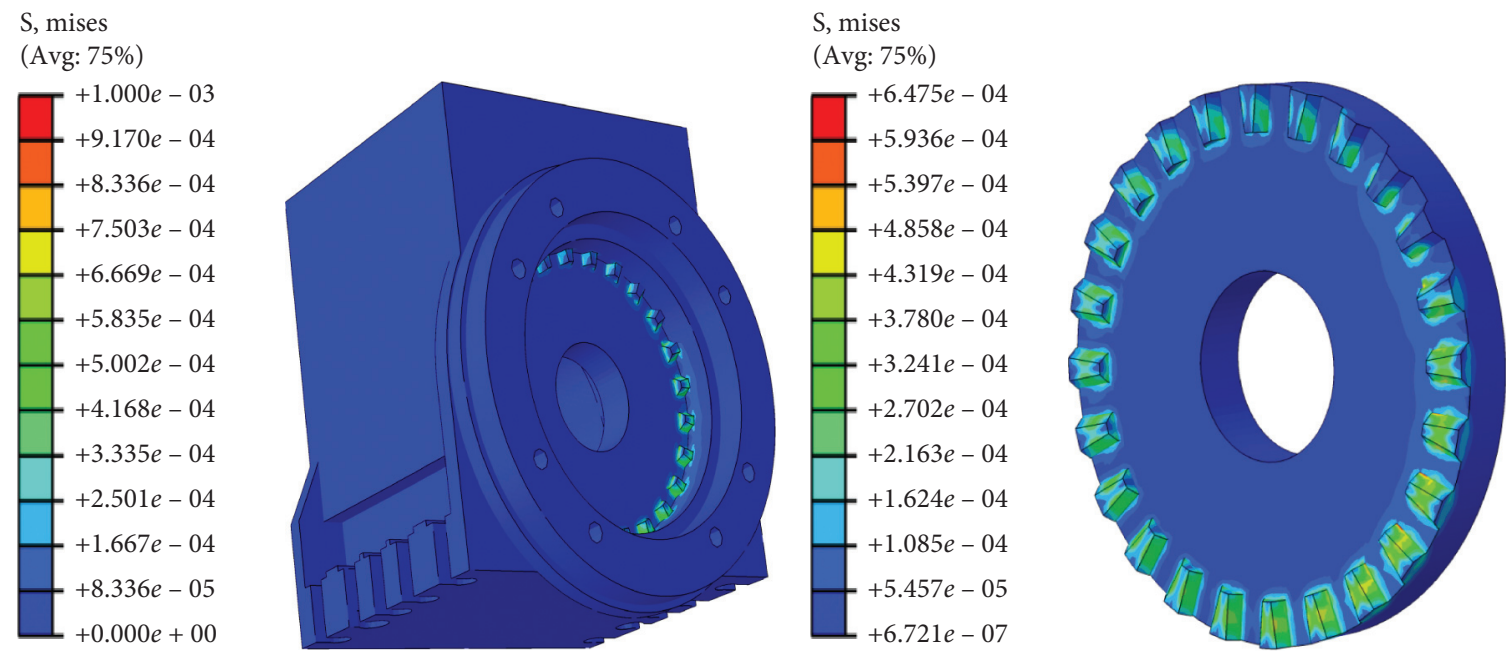

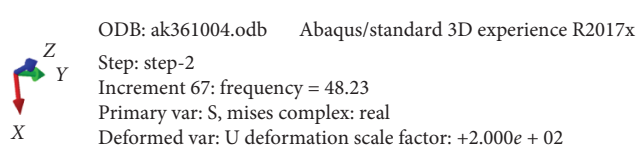

(a)
ODB: ak361004.odb Abaqus/standard 3D experience R2017x Step: step-2

Increment 67 : frequency $=48.23$

Primary var: $\mathrm{S}$, mises complex: real

Deformed var: $\mathrm{U}$ deformation scale factor: $+2.000 e+02$

FiguRE 18: Stress nephogram of area 1's fault simulation result.

transmitting shaft and transmitting base is not rigid and belongs to the clearance fit positioning. For paths 3 and 4, the spindle of the NC turret can make a limited movement, which will significantly affect the vibration energy transferred from the spindle to the NC turret shell in the $x_{-}, y_{\text {-, }}$ and $z$-directions. Compared with path 1 , the vibration energy flow of the NC turret shell caused by the dynamic cutting force in paths 2,3 , and 4 can be an order of magnitude larger than that of path 1 . Hence, the vibration energy flow of paths 2, 3, and 4 can be disregarded.

When the NC turret is subjected to dynamic cutting force, the influence caused by the poor meshing between the external fluted disc and different parts of the locking fluted disc is mainly studied. Therefore, the active subsystem could consist of the cutter rod, cutter holder, cutter head, internal fluted disc, and locking fluted disc in path 1 . Moreover, the passive subsystem is composed of the external fluted disc and NC turret shell. The excitation point functions as the dynamic cutting force acting on the cutter rod. The 24 pairs of teeth and 48 joint surfaces of the locking and external fluted discs were divided into 8 groups, with each group regarded as one coupling point. Additionally, 9 target points were chosen in 3 sensitive areas. The energy transmission diagram of path 1 is shown in Figure 20.
The 9 target points selected on the NC turret are shown in Figure 21. To calculate the path energy-keeping factor of area $i$ of the external fluted disc, the contact constraint in 1 area is retained, and the contact constraint in the other 7 areas is deleted. Keep the other constraints of the finite element model unchanged, and obtain the energy flow of the coupling point and 9 target points of the external fluted disc from the simulation results. Thereafter, the path energykeeping factor of the 9 target points in this area is calculated.

Given that the coupling point corresponds to several target points in this passive subsystem, the distribution rate is used to express the proportion of the energy transferred from the coupling point to each target point. The calculation results of the energy-keeping factor are shown in Table 2. The distribution rates of the energy flow transferred from the coupling points to the different target points are shown in Table 3.

The average distribution rate reflects the ability of the target points to obtain the energy flow of the coupling points. Standard deviation indicates the discrimination of the energy flow from different areas. The higher the standard deviation, the higher the discrimination. Table 3 shows that the average distribution rate and standard deviation of measurement points 1,4 , and 7 are both high. Therefore, target points 1, 4, and 7 are the sensitive measuring points of the poor meshing fault of the fluted disc. 


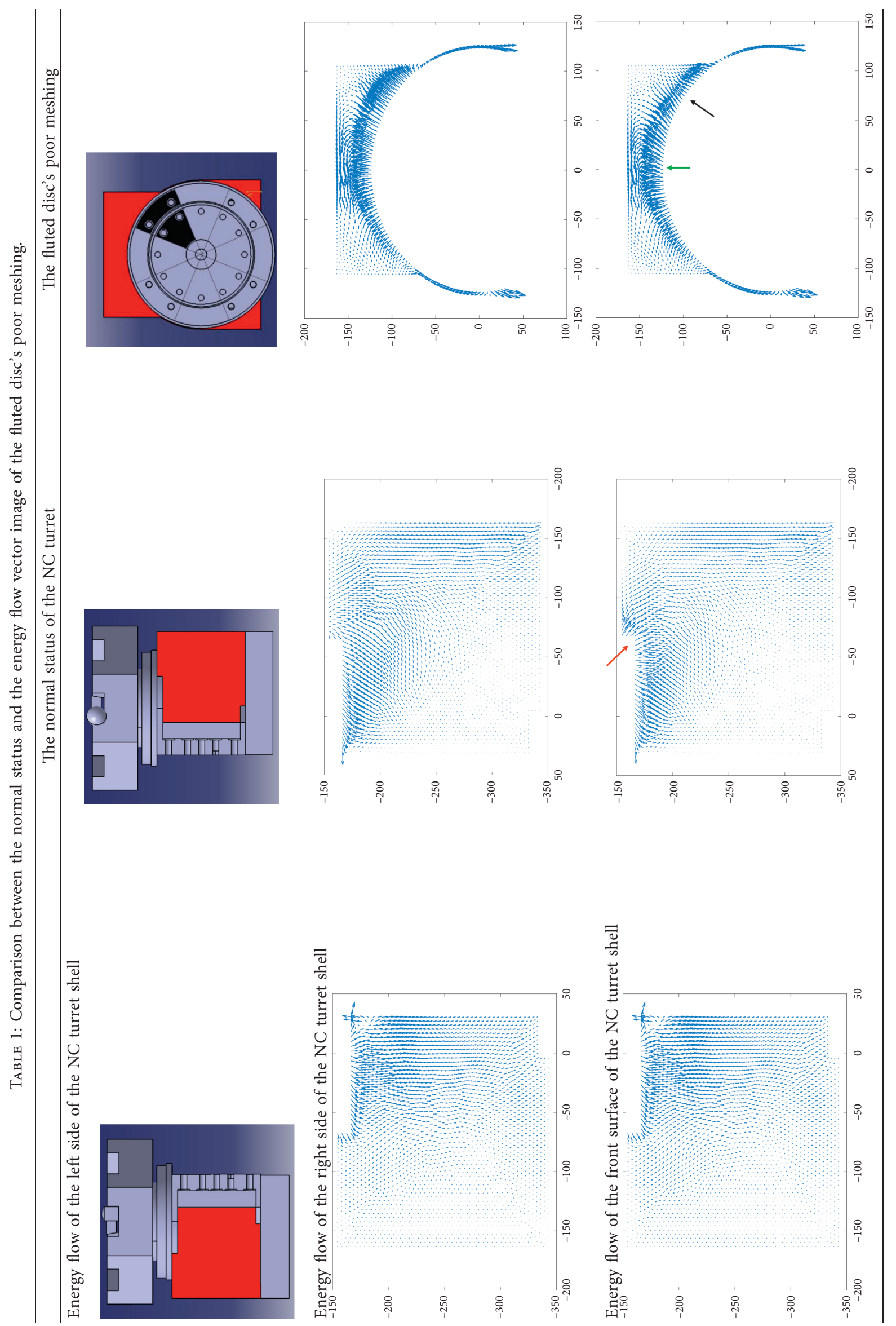



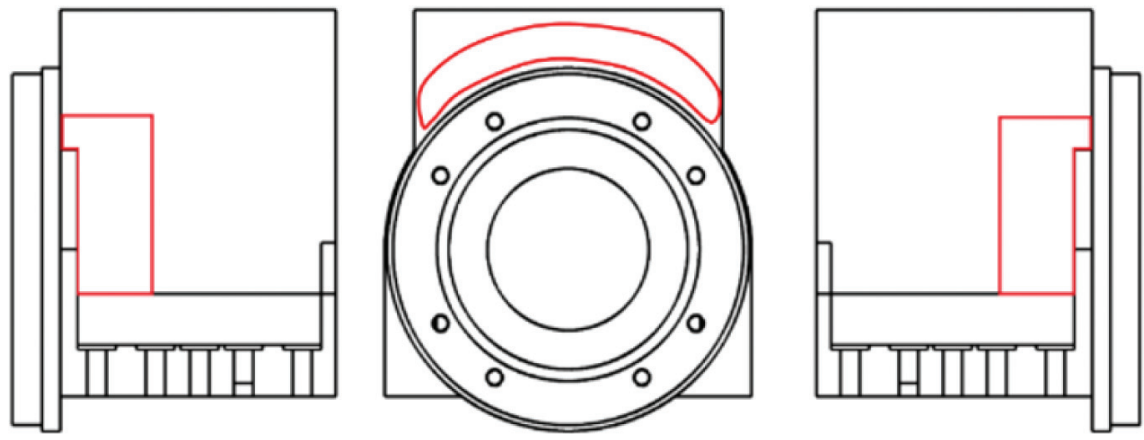

Figure 19: Sensitive areas of the fluted disc under vibration energy.

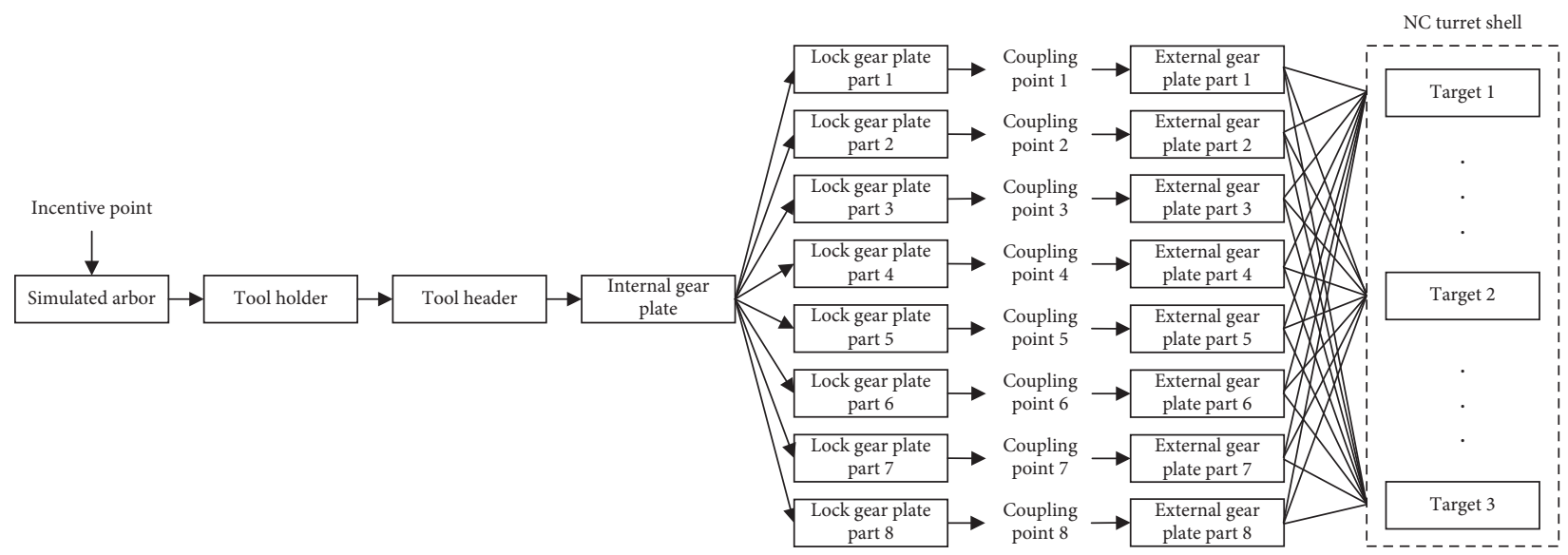

FIGURE 20: Energy transmission of path 1.
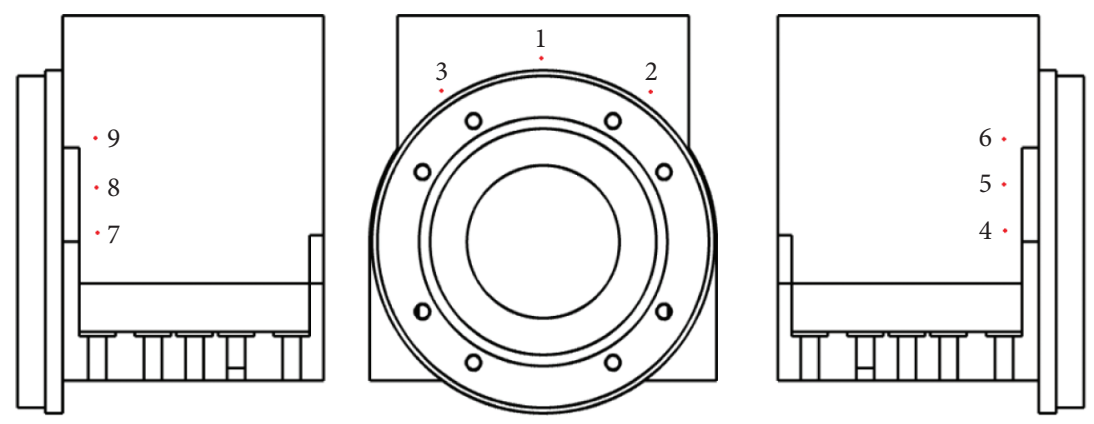

FIGURE 21: Location and number of the target points. 


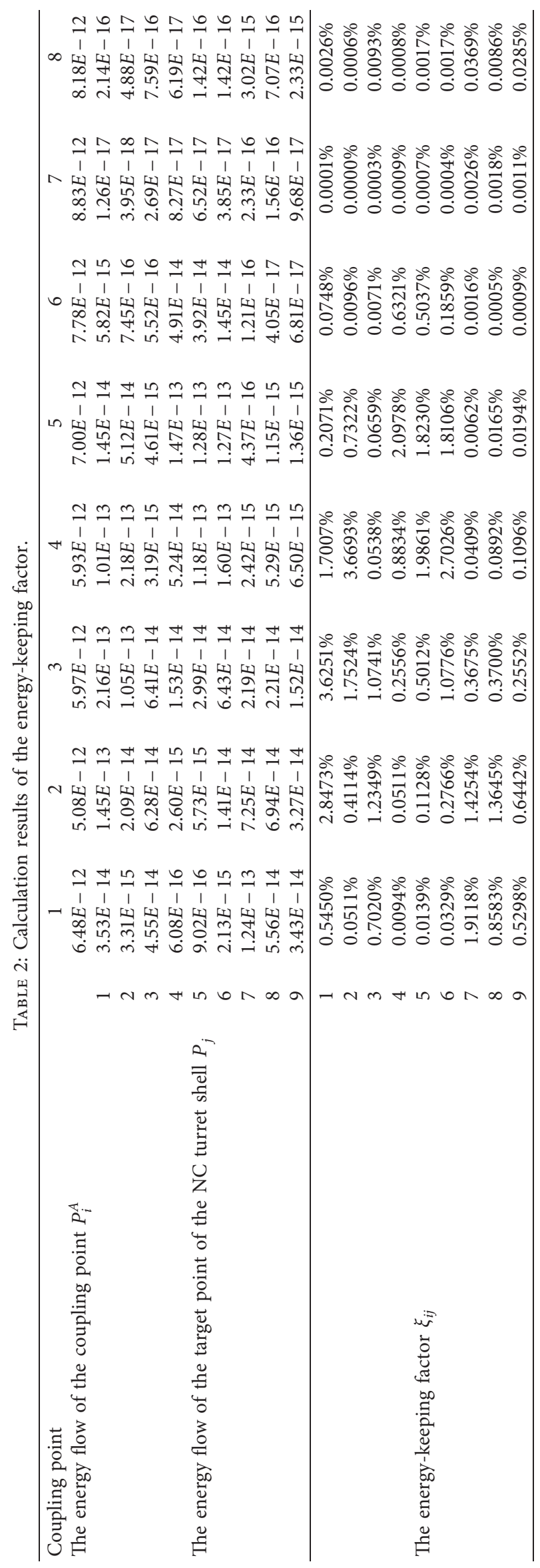


TABLE 3: Calculation results of the distribution rate of the energy flow.

\begin{tabular}{|c|c|c|c|c|c|c|c|c|c|c|}
\hline \multirow{2}{*}{$\begin{array}{l}\text { Target } \\
\text { point }\end{array}$} & \multicolumn{8}{|c|}{ Coupling point } & \multirow{2}{*}{$\begin{array}{c}\text { Average } \\
\text { distribution rare }\end{array}$} & \multirow{2}{*}{$\begin{array}{l}\text { Standard deviation of the } \\
\text { distribution rate }\end{array}$} \\
\hline & 1 & 2 & 3 & 4 & 5 & 6 & 7 & 8 & & \\
\hline 1 & $11.710 \%$ & $34.025 \%$ & $39.069 \%$ & $15.137 \%$ & $3.056 \%$ & $5.282 \%$ & $\begin{array}{c}1.764 \% \\
\%\end{array}$ & $4.210 \%$ & $14.282 \%$ & $14.524 \%$ \\
\hline 2 & $1.098 \%$ & $4.917 \%$ & $18.886 \%$ & $32.658 \%$ & $10.801 \%$ & $0.677 \%$ & $0.552 \%$ & $0.957 \%$ & $8.818 \%$ & $11.618 \%$ \\
\hline 3 & $15.082 \%$ & $14.757 \%$ & $11.576 \%$ & $0.479 \%$ & $0.972 \%$ & $0.501 \%$ & $3.755 \%$ & $14.906 \%$ & $7.754 \%$ & $6.928 \%$ \\
\hline 4 & $0.202 \%$ & $0.610 \%$ & $2.755 \%$ & $7.863 \%$ & $30.947 \%$ & $44.635 \%$ & $11.553 \%$ & $1.215 \%$ & $12.472 \%$ & $16.519 \%$ \\
\hline 5 & $0.299 \%$ & $1.348 \%$ & $5.402 \%$ & $17.677 \%$ & $26.892 \%$ & $35.568 \%$ & $9.107 \%$ & $2.794 \%$ & $12.386 \%$ & $13.067 \%$ \\
\hline 6 & $0.706 \%$ & $3.305 \%$ & $11.614 \%$ & $24.054 \%$ & $26.710 \%$ & $13.128 \%$ & $5.377 \%$ & $2.790 \%$ & $10.961 \%$ & $9.902 \%$ \\
\hline 7 & $41.077 \%$ & $17.033 \%$ & $3.960 \%$ & $0.364 \%$ & $0.092 \%$ & $0.110 \%$ & $32.573 \%$ & $59.247 \%$ & $19.307 \%$ & $22.622 \%$ \\
\hline 8 & $18.441 \%$ & $16.306 \%$ & $3.988 \%$ & $0.794 \%$ & $0.243 \%$ & $0.037 \%$ & $21.803 \%$ & $13.881 \%$ & $9.436 \%$ & $9.089 \%$ \\
\hline 9 & $11.384 \%$ & $7.699 \%$ & $2.750 \%$ & $0.975 \%$ & $0.287 \%$ & $0.062 \%$ & $13.516 \%$ & $0.001 \%$ & $4.584 \%$ & $5.504 \%$ \\
\hline
\end{tabular}

\section{Conclusion}

To solve the problem that some equipment fault measurement points are difficult to determine, this paper proposed a method for selecting the sensitive measuring points of the vibration fault based on energy flow and transfer path.

(1) Based on the energy flow theory of the shell, the energy flow is taken as the physical quantity to describe the energy transfer of the structure vibration. The energy flow at the coupling point and energy-keeping factor of each path are obtained, thereby enabling us to calculate the energy flow distribution rate of the coupling point to the different target points. Hence, a transfer path analysis method based on energy flow was established.

(2) By focusing on the NC turret, the simulation model of the finite element energy flow was established, and the vector image of the energy flow transfer path of the NC turret shell was created. Moreover, the energy flow test of the NC turret was conducted. Comparing the vector images of the experimental energy flow and simulation energy flow, the trend of the entire energy flow direction close to the simulation and the validity of the finite element energy flow simulation results are verified.

(3) By using the poor meshing fault of the fluted disc as a research object, three abnormal areas of the NC turret shell are identified by comparing and analyzing the energy flow vector image of the NC turret in the healthy and failure states. 9 target points are selected in the abnormal area to calculate the energy-keeping factor of the path from the abnormal area to the 9 target points and the distribution rates of each transfer path between the test points to the joint surfaces of the fluted disc. The calculation results indicated three sensitive measuring points that can accurately distinguish the typical failure modes.

This research combined energy flow theory and transfer path analysis method to determine the vibration fault sensitive measuring points of NC equipment. The proposed method is practical in the field of fault diagnosis and has broad application prospects.

\section{Data Availability}

The data used to support the findings of this study are included within the article.

\section{Conflicts of Interest}

The authors declare that they have no conflicts of interest.

\section{References}

[1] F. J. Fahy, "Statistical energy analysis: a critical overview," Philosophical Transactions of the Royal Society, vol. 346, no. 1681, pp. 431-447, 1994.

[2] A. Carcaterra, "An entropy formulation for the analysis of energy flow between mechanical resonators," Mechanical Systems and Signal Processing, vol. 16, no. 5, pp. 905-920, 2002.

[3] D. W. Miller and A. von Flotow, "A travelling wave approach to power flow in structural networks," Journal of Sound and Vibration, vol. 128, no. 1, pp. 145-162, 1989.

[4] D. U. Noiseux, "Measurement of power flow in uniform beams and plates," Journal of the Acoustical Society of America, vol. 47, no. 1, pp. 238-247, 2005.

[5] G. Jin, X. Ma, W. Wang, and Z. Liu, "An energy-based formulation for vibro-acoustic analysis of submerged submarine hull structures," Ocean Engineering, vol. 164, pp. 402-413, 2018.

[6] D. J. Mead, R. G. White, and X. M. Zhang, "Power transmission in a periodically supported infinite beam excited at a single point," Journal of Sound and Vibration, vol. 169, no. 4, pp. 558-561, 1994.

[7] M. Sheng, M. Wang, and W. Xing, "Mobility power flows between non-conservatively coupled structures with multipoint-connection," Mechanical Science and Technologu, vol. 20, no. 2, pp. 249-250, 2001, in Chinese.

[8] C. Simmons, "Structure-borne sound transmission through plate junctions and estimates of sea coupling loss factors using the finite element method," Journal of Sound and Vibration, vol. 144, no. 2, pp. 215-227, 1991.

[9] W. Zhang, F. Yu, and H. Liu, "Study on power flow characteristics of complicated system based upon the method combined mobility and mode synehesize," Modern Manufacturing, vol. 6, no. 9, pp. 6-8, 2007, in Chinese.

[10] Z. Chen, Z. Yang, Y. Gu, and S. Guo, "An energy flow model for high-frequency vibration analysis of two-dimensional panels in supersonic airflow," Applied Mathematical Modelling, vol. 76, pp. 495-512, 2019. 
[11] K. S. Oh, H. K. Kim, K. S. Yi, K. E. Ko, P. Y. Kim, and J. H. Seo, "Energy flow analysis of working and driving system of a wheel loader," Journal of The Korean Society for Fluid Power and Construction Equipments, vol. 11, no. 2, pp. 22-29, 2014.

[12] H.-W. Kwon, S.-Y. Hong, and J.-H. Song, "Energy flow models for underwater radiation noise prediction in mediumto-high-frequency ranges," Proceedings of the Institution of Mechanical Engineers, Part M: Journal of Engineering for the Maritime Environment, vol. 230, no. 2, pp. 404-416, 2016.

[13] M. V. V. D. Seijs, D. D. Klerk, and D. J. Rixen, "General framework for transmission path analysis: history, theory and classification of techniques," Mechanical Systems and Signal Processing, vol. 68-69, pp. 217-244, 2015.

[14] Z. Wei and Z. Yi-Min, "Reliability analysis of random vibration transmission path systems," Mathematical Problems in Engineering, vol. 2017, Article ID 6814547, 6 pages, 2017.

[15] K. Janssens, P. Gajdatsy, L. Gielen et al., "OPAX: a new transfer path analysis method based on parametric load models," Mechanical Systems and Signal Processing, vol. 25, no. 4, pp. 1321-1338, 2011.

[16] Q. Li, K. Xue, and Y. Li, "Study on power flow characteristics of a double half shaft system," Journal of Harbin Engineering University, vol. 32, no. 9, pp. 1163-1167, 2011, in Chinese.

[17] W. Huang, H. Sun, H. Dou, and W. Wang, "Study of transfer path of wind turbine gearbox fault vibration signal based on power flow finite element method," Advances in Mechanical Engineering, vol. 9, no. 5, Article ID 168781401770389, 2017.

[18] H. M. Zhao, D. Y. Li, W. Deng et al., "Research on vibration suppression method of alternating current motor based on fractional order control strategy," Proceedings of the Institution of Mechanical Engineers, Part E: Journal of Process Mechanical Engineering, vol. 231, no. 4, pp. 786-799, 2017.

[19] H. Zhao, S. Zuo, M. Hou et al., "A novel adaptive signal processing method based on enhanced empirical wavelet transform technology," Sensors, vol. 18, no. 10, p. 3323, 2018.

[20] X. D. Xu, H. P. Lee, and C. Lu, "The structural intensities of composite plates with a hole," Composite Structures, vol. 65, no. 3-4, pp. 493-498, 2004.

[21] W. Zhao, Y.-M. Zhang, and W.-Z. Feng, "Path contribution analysis of vibration transfer path systems," Mathematical Problems in Engineering, vol. 2019, Article ID 1458149, 14 pages, 2019.

[22] Z. Sun, H. Qin, C. Wang, and J. Sun, "Error analysis on measurement of vibration intensty based on dual transducers," Journal of Vibration Engineering, vol. 9, no. 3, pp. 253-259, 1996, in Chinese. 